\title{
Características de la limitación de terapia de soporte vital en pacientes fallecidos en unidades de medicina intensiva
}

\author{
Bernardina Frache* ${ }^{\star}$, Eduardo Moreirałs, Agustín Carámbula*, Carlos Pan`, \\ Marcelo Barbatoł, Pedro Azlugaray ${ }^{* \star * *+}$, Gastón Burghi*
}

\section{Resumen}

Introducción: la limitación de terapias de soporte vital (LTSV) es una práctica habitual y éticamente aceptada. La misma se enmarca en el concepto de adecuación del esfuerzo terapéutico.

Objetivo: determinar la frecuencia, las directivas de implementación y los factores asociados a LSTV en los pacientes fallecidos en una unidad de cuidados intensivos (UCl). Conocer la frecuencia y características del registro de este proceso en la historia clínica.

Diseño: estudio observacional multicéntrico de corte transversal.

Ámbito: se analizaron las historias clínicas de ocho UCI polivalentes públicas y privadas de Uruguay.

Pacientes: se incluyeron todos los pacientes mayores de 18 años fallecidos en un período de seis meses consecutivos en el año 2011.

Intervenciones: análisis de las características de los pacientes mediante revisión de historias clínicas.

Resultados: analizamos 210 pacientes fallecidos. Se identificó LSTV en 63 (30\%). El 75\% de las decisiones consistió en la retirada de medidas terapéuticas. Los factores independientemente asociados a LTSV fueron: mayor edad [OR 1,04 (IC 95\% 1,01-1,07), $p=0$,001], mayor estadía en UCI [OR 1,06 (IC 95\% 1,02 - 1,11), $p=0,001$ ] y limitación para las actividades de la vida diaria [OR 4,65 (IC 95\% 1,1-19,6), p=0,035]. El 70\% de las directivas LTSV fueron registradas en la historia clínica. Los soportes vitales más frecuentemente retirados fueron: nutrición parenteral $(100 \%)$, vasopresores $(79 \%)$, nutrición enteral (64\%) y antimicrobianos (52\%). La asistencia ventilatoria mecánica invasiva se retiró en el $33 \%$ de los casos.

Conclusiones: un tercio de los pacientes fallece con LTSV. Este tipo de decisiones se asocian a mala calidad de vida previa, mayor edad y mayor estadía en UCI. Un tercio de las decisiones de LTSV no son registradas.

Palabras clave: Privación de tratamiento

Key words: Withholding treatment

Unidades de cuidados intensivos

Intensive care units

Cuidado terminal

Terminal care

Toma de decisiones

Decision making

\footnotetext{
* Médico Intensivista. UCI Sanatorio Americano. Montevideo, Uruguay.

$†$ Médico Intensivista. UCI Sanatorio CAMOC. Colonia, Uruguay.

$\ddagger$ Médico Intensivista. UCI Hospital Maciel, ASSE. Montevideo, Uruguay.

$\S$ Medico Intensivista. UCI Sanatorio CASMER. Rivera, Uruguay.

II Médico Intensivista. UCI Sanatorio CAAMEPA. Canelones, Uruguay.

** Médico Intensivista. UCI Sanatorio ORAMECO. Colonia, Uruguay.

†† Médico Intensivista. UCI Sanatorio CRAMI. Las Piedras, Uruguay.

Unidad de Cuidados Intensivos Hospital Maciel.

Unidad de Cuidados Intensivos Hospital Policial.

Unidad de Cuidados Intensivos Sanatorio Americano.

Unidad de Cuidados Intensivos Sanatorio CAMOC.

Unidad de Cuidados Intensivos Sanatorio CAAMEPA.

Unidad de Cuidados Intensivos Sanatorio CRAMI.

Unidad de Cuidados Intensivos Sanatorio ORAMECO.

Unidad de Cuidados Intensivos Sanatorio CASMER.

Aprobado por el Comité de Ética del Centro Asistencial Médico de Rivera.

Los autores declaran no tener conflicto de intereses.

Correspondencia: Dr. Eduardo Moreira. Hospital Maciel. 25 de Mayo 174. Montevideo, Uruguay.

Correo electrónico: dreduardo.moreira@gmail.com
} 


\section{Introducción}

El envejecimiento de la población y el aumento de la sobrevida de los pacientes con patologías crónicas han conducido al ingreso a unidad de cuidados intensivos (UCI) de pacientes cada vez más añosos y con un mayor número de comorbilidades. Este cambio en el perfil de los pacientes de la UCI se acompañan del lógico cuestionamiento respecto a cuáles son los pacientes que realmente se benefician de la medicina intensiva, y en cuáles estamos simplemente realizando tratamientos invasivos y costosos sin perseguir un claro objetivo. En este sentido, Walling A y colaboradores reportan que en un año de estudio, el 56\% de los 496 pacientes fallecidos en su unidad presentaban una patología en etapa terminal ${ }^{(1)}$. Estas situaciones, entre otras, llevan a plantear la adecuación del esfuerzo terapéutico (AET) y la limitación de terapias de soporte vital (LTSV), donde algunos tratamientos no se instauran o se suspenden, cambiando el enfoque de cuidados tendientes a priorizar el confort del paciente ${ }^{(2)}$.

La AET y la indicación de LTSV vienen siendo un elemento de estudio desde hace varias décadas ${ }^{(3-6)}$. Si bien existen diferencias entre los distintos países en cuanto al tipo de paciente en que se plantea la limitación de determinados tratamientos y en la forma de llevar a cabo este proceso, todos los países, con sus diferencias culturales, limitan el acceso o retiran determinados tratamientos ${ }^{(7-9)}$. Recientemente, Lobo y colaboradores en su estudio que incluyó 9.524 ingresos a UCI de todo el mundo, pusieron en evidencia significativas diferencias en la toma de decisiones de AET en los diferentes continentes. En este sentido, mientras que se limitaban o suspendían terapéuticas en el $3 \%$ de los pacientes en el sur asiático, en Oceanía estas decisiones alcanzan el 30\% de los ingresos. Asimismo, los países con mayores ingresos toman más frecuentemente este tipo de decisiones al compararlos con aquellos de menores recursos económicos ${ }^{(10)}$.

Así como existen diferencias en la frecuencia y forma de llevar adelante la limitación de determinados tratamientos, también se presentan diferencias en la comunicación de estas decisiones, en el grado de participación de la familia y en la forma de registrarlas en la historia clínica.

Los objetivos de este estudio son determinar la frecuencia y los factores asociados a la LTSV entre los pacientes fallecidos en UCI, conocer la forma de documentar este proceso y las directivas de adecuación de la terapéutica.

\section{Metodología}

Se llevó a cabo un estudio retrospectivo multicéntrico en ocho UCI polivalentes médico-quirúrgicas de Uru- guay. Las unidades participantes fueron seis unidades privadas (una de Montevideo y cinco del interior) y dos unidades públicas de Montevideo. Se solicitó al departamento de registros médicos de las ocho instituciones participantes las historias clínicas de todos los pacientes fallecidos en la UCI de adultos desde el 1 de julio hasta el 31 de diciembre del año 2011, a dos años de la promulgación de la Ley N. ${ }^{\circ} 18473$, publicada en el Diario Oficial el 21 abril de 2009, N. ${ }^{\circ} 27714$ de Voluntad Anticipada. Se excluyeron del estudio los pacientes fallecidos menores de 18 años.

Las historias fueron analizadas por médicos ajenos al equipo de cada unidad, de forma que los médicos de una unidad no analizaran las historias de su propio centro.

Mediante un cuestionario estandarizado se recolectaron informaciones de las características de los pacientes, la existencia de una decisión de LSTV, la presencia de un registro médico de la misma, de las personas participantes en la decisión y de la forma de implementar la LSTV en cada caso (tratamientos retirados e instaurados).

Se consideró LSTV cuando se retiró cualquier soporte vital o tratamiento fuera de un contexto de mejoría de la función vital referida. En el caso de la ventilación mecánica invasiva se consideró su limitación cuando se descendió la fracción inspirada de oxígeno, se colocaba en modalidad espontánea como una medida alterantiva al tubo en $\mathrm{T}$, o cuando el paciente se colocaba en tubo en $\mathrm{T}$ sin un adecuado valor de oxemia o situación clínica que lo justificara.

La constatación de LTSV estuvo determinada por el registro médico de la misma en la historia, o mediante el análisis de la planilla de prescripciones y controles de enfermería donde se evidenciaba una retirada o no instauración de tratamientos de soporte vital, aunque no se registrara la indicación explícitamente.

\section{Análisis estadístico}

Para llevar adelante el análisis de los resultados se utilizó el paquete estadístico SPSS 19. Las comparaciones de variables categóricas se hicieron con las pruebas exactas de Fisher y de chi cuadrado (con la corrección de Yates cuando esté indicado). Las variables continuas se presentan en la forma de mediana (intervalo intercuartil) y se compararon con la prueba t-Student o la prueba U de Mann-Whitney Rank-sum, según esté indicado. Para determinar las variables independientemente asociadas a las decisiones de AET se incluyeron en un modelo de análisis multivariado por regresión logística aquellas variables que presentaron en el análisis bivariado una $\mathrm{p}<0,2$. En todos los análisis se adoptó como nivel de significancia estadística un $\mathrm{p}$ valor $<0,05$ bilateral. 


\section{Resultados}

\section{Características generales}

Se cumplieron los criterios de inclusión en 210 casos (figura 1). El 55\% de estos fueron de sexo masculino, con una edad de 67 años (53,5-76). Se llevó a cabo LSTV en 63 pacientes (30\%). El 75\% de las decisiones consistió en la retirada de medidas terapéuticas, en el $23 \%$ se decidió no incrementar las medidas ya dispuestas y solo un paciente ( $2 \%$ ) tenía una directiva anticipada registrada de no reanimación cardiopulmonar. Las características generales de la población se presentan en la tabla 1.

\section{Decisiones de LTSV de acuerdo con las características de los pacientes}

Se encontró registró de la LTSV a los 6 (1-10) días del ingreso a la UCI. El porcentaje de fallecidos con limitación de alguna terapéutica se incrementó a medida que transcurrió el tiempo de estadía en la UCI (figura 2). Los factores asociados a LTSV por análisis bivariado se presentan en la tabla 2. El análisis multivariado identificó que los factores que estuvieron independientemente asociados a LSTV fueron mayor edad [OR 1,04 (IC 95\% $1,01-1,07), p=0,001]$, mayor estadía en UCI [OR 1,06 (IC $95 \% 1,02-1,11$ ), $\mathrm{p}=0,001]$ y presencia de limitación funcional previa [OR 4,65 (IC 95\% 1,1-19,6), $\mathrm{p}=0,035]$ (tabla 3 ).

\section{Documentación y comunicación de las decisiones de LTSV}

El 70\% de los pacientes que fallecieron luego de una decisión de LSTV presentaron registro de esta directiva por parte de los médicos en la historia clínica. En 13\% se describe quienes decidieron dicha adecuación terapéutica y en 39\% quedó registro escrito de la comunicación a la familia de la conducta adoptada. En el 6\% existió documentación de la participación de la familia en la decisión.

\section{Directivas de la LTSV}

Los soportes vitales más frecuentemente retirados fueron: nutrición parenteral (100\%), vasopresores $(79 \%)$, nutrición enteral (64\%), antimicrobianos $(52 \%)$ y asistencia ventilatoria mecánica invasiva (33\%).

De los pacientes a los cuales se les retiró el soporte ventilatorio, a $25 \%$ (16 pacientes) se los colocó en tubo en T. En los restantes pacientes se mantuvo la ventilación mecánica, pero se descendieron o modificaron determinados parámetros (fracción inspirada de oxígeno, retiro de una modalidad controlada). No se realizaron extubaciones terminales (tabla 3 ).

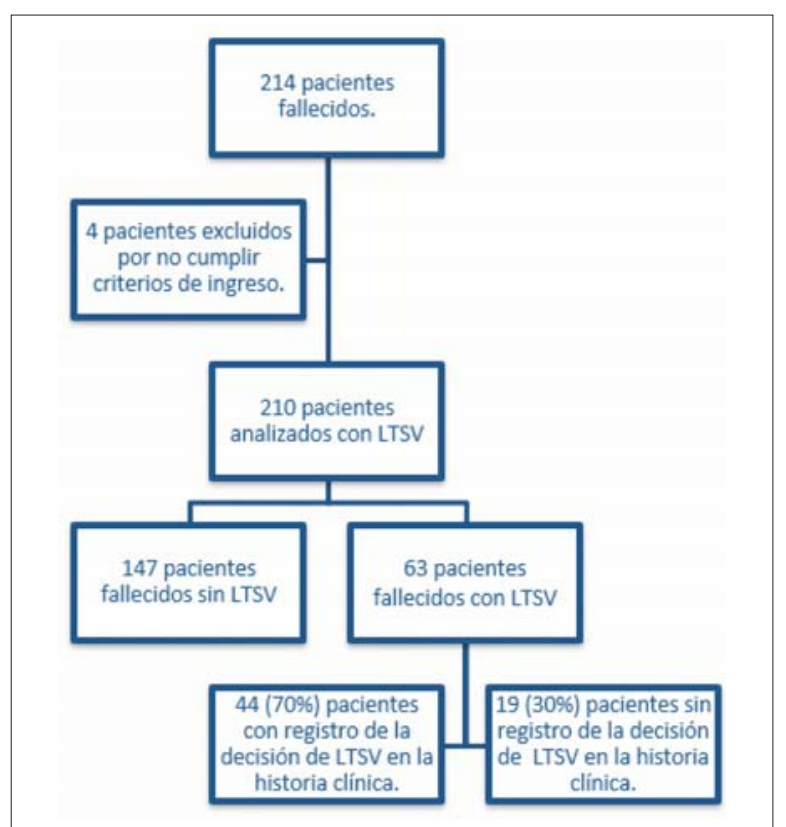

Figura 1. Diagrama de selección de pacientes.

\section{Discusión}

La LTSV es una práctica habitual en todas partes del mundo ${ }^{(10,11)}$. Las diferentes culturas, religiones y legislaciones generan diferentes formas de llevar a cabo la puesta en marcha de este proceso. Estas diferencias existen inclusive entre especialistas de un mismo país. En este sentido, el estudio realizado por Yaguchi A y colaboradores, que evaluó las conductas de los intensivistas frente a una serie de situaciones hipotéticas, puso en evidencia las diferencias existentes entre diferentes culturas a la hora de tomar decisiones al final de la vida, pero también entre especialistas de un mismo país ${ }^{(7)}$.

En nuestro estudio, la tercera parte de los pacientes fallecidos en UCI lo hacen luego de una decisión de LTSV. La frecuencia de este tipo de decisiones es muy variable entre los diferentes estudios. Lobo y colaboradores, en su reciente estudio, encontraron diferencias entre las distintas regiones al evaluar la frecuencia de $\mathrm{LTSV}^{(10)}$. En este estudio la frecuencia de pacientes fallecidos luego de una decisión de limitación o retiro de los tratamientos varió entre $10 \%$ en el sur de Asia y $65 \%$ en Norteamérica. En América del Sur este estudio encontró que el $25 \%$ de los pacientes fallecidos en un hospital lo hacían luego de no iniciar o suspender algún tratamiento. Sin embargo, el estudio llevado a cabo por Piva y colaboradores en Brasil evidencia que el $86 \%$ de los pacientes adultos y el $43 \%$ de los niños fallecidos en UCI presentaron una decisión de $\mathrm{AET}^{(12)}$.

En nuestra población los pacientes más añosos presentaron más frecuentemente registro de una decisión de 
Tabla 1. Características generales de la población analizada.

\begin{tabular}{|c|c|}
\hline Características de la población. $N=210$ & $\begin{array}{c}\% \text { (n) o mediana } \\
\text { (25\%-75\%) }\end{array}$ \\
\hline Edad & $67(53,5-76)$ \\
\hline Sexo femenino & $45 \%(95)$ \\
\hline Días de internación previo al ingreso a $\mathrm{UCl}$ & $0(0-1)$ \\
\hline Días de internación en UCl & $4(2-8,25)$ \\
\hline \multicolumn{2}{|l|}{ Comorbilidades } \\
\hline EPOC & $26 \%(54)$ \\
\hline Diabetes & $20 \%(42)$ \\
\hline Cardiopatía & $21 \%(45)$ \\
\hline Cáncer & $9 \%(19)$ \\
\hline Sida & $2 \%(4)$ \\
\hline Presencia de más de dos comorbilidades & $16 \%(34)$ \\
\hline \multicolumn{2}{|l|}{ Causa de ingreso } \\
\hline Patología respiratoria & $26 \%(55)$ \\
\hline Patología cardiovascular & $16 \%(33)$ \\
\hline Patología neurológica & $22 \%(46)$ \\
\hline Politraumatizados & $11,5 \%(24)$ \\
\hline Sepsis & $11,5 \%(24)$ \\
\hline Otras & $13 \%(26)$ \\
\hline SAPS ॥ & $58(46-75)$ \\
\hline Necesidad de vasopresores al ingreso & $94 \%(198)$ \\
\hline \multicolumn{2}{|l|}{ Necesidad de ventilación mecánica } \\
\hline VMI (ventilación mecánica invasiva) & $91 \%(191)$ \\
\hline VMNI (ventilación mecánica no invasiva) & $2 \%(5)$ \\
\hline Terapia de reemplazo renal & $4 \%(8)$ \\
\hline Decisión de LTSV & $30 \%(63)$ \\
\hline \multicolumn{2}{|l|}{ Tipo de directiva } \\
\hline Retirada de medidas terapéuticas & $75 \%(48 / 63)$ \\
\hline No incremento de las medidas instauradas & $25 \%(16 / 63)$ \\
\hline \multicolumn{2}{|l|}{ Frecuencia de LTSV de acuerdo con el centro } \\
\hline UCI 1 & $24 / 54(44 \%)$ \\
\hline $\mathrm{UCl} 2$ & $6 / 31(19 \%)$ \\
\hline UCI 3 & $3 / 7(43 \%)$ \\
\hline UCl 4 & $3 / 26(12 \%)$ \\
\hline UCI 5 & $10 / 46(22 \%)$ \\
\hline $\mathrm{UCl} 6$ & $3 / 14(21 \%)$ \\
\hline UCl 7 & $12 / 25(48 \%)$ \\
\hline UCl 8 & $2 / 7(29 \%)$ \\
\hline
\end{tabular}

AET previo a su fallecimiento en UCI. En este mismo sentido, el estudio realizado por el Southern Cone End-of-Life Care Workgroup, que incluyó la opinión de 62 trabajadores de UCI de Uruguay (52 médicos intensivistas), evidenció que la edad del paciente fue uno los dos factores mayormente asociados a la toma de decisiones de $\mathrm{AET}^{(13)}$. El estudio llevado a cabo por Azoualy y colaboradores en el cual analizaron datos de 282 UCI de diferentes continentes, encontraron que las comorbilidades y la edad se asociaban a la toma de decisiones de limitación de la terapéutica ${ }^{(14)}$. Más recientemente, Lobo y colaboradores, en su estudio que incluyó UCI de todo el mundo, encontraron que una mayor severidad al ingreso, la presencia de más de dos disfunciones orgánicas, las comorbilidades severas y los ingresos por patologías médicas y por trauma se asociaron más frecuentemente con la toma de decisiones de adecuación de la terapéutica ${ }^{(10)}$.

Requerir asistencia para el desarrollo de las actividades básicas de la vida diaria se asocia con las decisiones de LTSV en nuestro estudio. La calidad de vida es un factor frecuentemente tenido en cuenta a la hora de tomar una decisión de AET y LTSV. En este sentido, Sprung y colaboradores, entre 1.899 encuestados (médicos, nurses, pacientes y familiares), encontraron que para el $75 \%$ de ellos, la calidad de vida fue el factor priorizado a la hora de tomar una decisión de este tipo ${ }^{(15)}$. En el estudio del Southern Cone End-of-Life Care Workgroup la calidad de vida previa era tomada en cuenta por más del $60 \%$ de los profesionales uruguayos que intervinieron de esta encuesta ${ }^{(13)}$. Recientemente, Blazquez y colaboradores, en un estudio prospectivo llevado a cabo en 11 unidades de España, encontraron que la calidad de vida previa era el factor con mayor impacto en las decisiones de LTSV en las primeras 48 horas del ingreso a $\mathrm{UCI}^{(16)}$.

Nuestro estudio muestra que una mayor estadía en UCI se relaciona con un incremento de las decisiones. Si bien la edad, las comorbilidades y la calidad de vida previas son factores asociados a la LTSV, existen otros factores asociados a este proceso. En este sentido, el fracaso de los tratamientos también se asocia a decisiones de LTSV. La futilidad es frecuentemente una razón para adecuar los tratamientos. Este aspecto ha sido evaluado en diversos estudios ${ }^{(13,14)}$. El sentimiento de futilidad y el fracaso de las terapéuticas instauradas podrían explicar la asociación entre mayor estadía en UCI y decisiones de LTSV.

El registro de datos clínicos y paraclínicos es muy variable en los distintos estudios y la LTSV no escapa a esta realidad ${ }^{(17)}$. En nuestro estudio, a pesar de la promulgación de la ley de voluntad anticipada, la documentación de las decisiones se realizó en el 68\% de los casos, 
Tabla 2. Características de los pacientes que están asociadas a la decisión de LTSV.

\begin{tabular}{|c|c|c|c|}
\hline & Fallecimiento con LTSV & Fallecimiento $\sin L T S V$ & $P$ \\
\hline Edad & $70,5(62,75-82,25)$ & $63(50-75)$ & 0,001 \\
\hline Días previos al ingreso a $\mathrm{UCl}$ & $0(0-2)$ & $0(0-1)$ & 0,158 \\
\hline Estadía en UCl & $7(3-11)$ & $3(1-8)$ & 0,001 \\
\hline Limitación para ABVD & $14 \%(9)$ & $2 \%(3)$ & 0,000 \\
\hline \multicolumn{4}{|l|}{ Comorbilidades } \\
\hline EPOC & $36 \%(23)$ & $21 \%(31)$ & 0,021 \\
\hline Diabetes & $29 \%(18)$ & $16 \%(24)$ & 0,045 \\
\hline Cardiopatía & $21 \%(13)$ & $22 \%(32)$ & 0,85 \\
\hline Cáncer & $6 \%(4)$ & $10 \%(15)$ & 0,37 \\
\hline Sida & $3 \%(2)$ & $1 \%(2)$ & 0,37 \\
\hline Presencia de más de dos comorbilidades & $21 \%(13)$ & $14 \%(21)$ & 0,23 \\
\hline SAPS II & $64(50,5-75)$ & $55(45-77)$ & 0,38 \\
\hline \multicolumn{4}{|l|}{ Diagnóstico al ingreso } \\
\hline Patología respiratoria & $30 \%(19)$ & $25 \%(36)$ & 0,59 \\
\hline Patología cardiovascular & $19 \%(12)$ & $14 \%(21)$ & \\
\hline Patología neurológica & $24 \%(15)$ & $21 \%(31)$ & \\
\hline Politraumatizado & $8 \%(5)$ & $13 \%(19)$ & \\
\hline Sepsis & $11 \%(7)$ & $12 \%(17)$ & \\
\hline Otros & $8 \%(5)$ & $14 \%(21)$ & \\
\hline
\end{tabular}

Tabla 3. Factores independientemente asociados a AET por análisis multivariado.

\begin{tabular}{lccc}
\hline & OR & IC 95\% & P \\
\hline Edad (por cada año) & 1,04 & $1,01-1,07$ & 0,001 \\
Días en UCI (por cada día) & 1,06 & $1,02-1,11$ & 0,001 \\
Limitación para ABVD & 4,65 & $1,1-19,6$ & 0,035 \\
EPOC & 1,50 & $0,71-3,19$ & 0,28 \\
Diabetes & 1,80 & $0,81-3,9$ & 0,14 \\
\hline
\end{tabular}

habitualmente no detalla quiénes participaron de las mismas, ni tampoco relata la discusión y comunicación de este proceso con la familia. Piva y colaboradores, en su estudio desarrollado en 10 UCI de Brasil, evaluaron 1.053 fallecimientos ( $40 \%$ en niños), las decisiones de limitación de la terapéutica fueron registradas en 52,6\% de los pacientes pediátricos y en $33,7 \%$ de los adultos ${ }^{(12)}$.
El subregistro de las decisiones de LTSV puede estar vinculado al desconocimiento del marco legal referido a la LTSV. Del desconocimiento de las normas vigentes surgen temores por reclamaciones legales. Este aspecto fue puesto en evidencia en el estudio del Southern Cone End-of-Life Care Workgroup ${ }^{(13)}$. 


\begin{tabular}{|lc|}
\hline \multicolumn{2}{|l|}{ Tabla 4. Directivas de LTSV. } \\
\hline Medidas terapéuticas retiradas & $\%(n)$ \\
\hline Nutrición parenteral & $100 \%(3 / 3)$ \\
Vasopresores & $79 \%(38 / 48)$ \\
Nutrición enteral & $64 \%(23 / 36)$ \\
Antimicrobianos & $52 \%(14 / 27)$ \\
Ventilación mecánica & $33 \%(17 / 51)$ \\
Sedación/analgesia & $23 \%(14 / 61)$ \\
Protección gástrica & $16 \%(10 / 64)$ \\
Reposición hidroelectrolítica & $6 \%(4 / 64)$ \\
\hline
\end{tabular}

Por su parte, el estudio de Spronk y colaboradores, que evaluó 293 fallecidos en dos UCI de Alemania, también encontró deficiencias en el registro de la limitación de los tratamientos. Estos autores lo asocian a la sobrecarga de trabajo en las unidades y a la falta de entrenamiento en el proceso de limitación del esfuerzo terapéutico ${ }^{(18)}$. Estos mismos factores podrían estar en juego en la falta de registro evidenciado en nuestro estudio.

El tipo de tratamiento retirado cuando se decide una adecuación del esfuerzo terapéutico es variable en los diversos estudios. El estudio de Mani y colaboradores muestra claramente cómo los pacientes fallecidos luego de una LTSV son sometidos a menos intervenciones y estudios diagnósticos que el resto de los pacientes ${ }^{(19)}$.

En nuestro estudio, a la tercera parte de los pacientes fallecidos se decidió el retiro de la ventilación mecánica, ya sea colocando al paciente en tubo en t o alterando los parámetros ventilatorios, sin existencia de casos de extubación terminal. Contrariamente a nuestros hallazgos, la extubación terminal es una práctica frecuente en diversos sitios. El estudio desarrollado por Long y colaboradores, analizando 330 pacientes fallecidos luego de una decisión de LTSV, mostró que el 74\% de los pacientes eran extubados previo a su fallecimiento ${ }^{(20)}$. Por su parte, Grestel y colaboradores encontraron que la extubación terminal se relaciona con un incremento de la satisfacción familiar ${ }^{(21)}$.

El estudio presenta una serie de limitaciones. Por ser un estudio retrospectivo con revisión de historias clínicas, no se analizan características de los profesionales actuantes, tasa de ocupación de la UCI o relación paciente-intensivista. Si bien este es un estudio muticéntrico, no lo podemos considerar representativo de la realidad de todo el país. Al analizar exclusivamente los pacientes fallecidos en la UCI, no podemos conocer la frecuencia en que se retiran o no se comienzan determina- dos tratamientos en la totalidad de los pacientes en los que se decide adecuar la terapéutica.

Existen factores posiblemente asociados a LTSV, como el agravio neurológico o la persistencia de disfunción multiorgánica que no fueron analizados.

La ausencia de registros de LTSV en la historia clínica no nos permite asegurar que no existió comunicación de las decisiones a la familia, ni tampoco la falta de participación de la misma en este proceso.

\section{Conclusiones}

En la muestra, una de cada tres muertes se produjo luego del retiro o no inicio de una o más medidas de soporte vital. Identificamos algunos factores asociados a este tipo de decisiones, donde la edad, la calidad de vida y las comorbilidades toman un especial peso. Existen deficiencias en la calidad del registro de la LTSV.

\section{Abstract}

Introduction: withdraw and withhold life support therapies is an usual and ethically accepted practice.

Objectives: to determine the frequency, the directives and factors associated with withdrawing and withholding life support therapies in patients who died in ICU. Determine the frequency and characteristics of the record of this process in clinical history.

Design: multicentric observational study of cross section.

Setting: clinical histories of 8 public and private mixed ICUs in Uruguay were analyzed.

Patients: all patients over 18 years who died in a period of 6 consecutive months in 2011 were included.

Interventions: analysis of the characteristics of patients through review of clinical histories.

Results: we analyzed 210 patients who died. Withdrawing or withholding was identified in 63 (30\%). 75\% of the decisions consisted in withdraw therapeutic measures. Factors independently associated to withdrowing or withholding therapies were: an older age [OR 1.04 (95\% CI 1.01-1.07), $\mathrm{p}=0.001$ ], a longer stay in ICU [OR $1.06(95 \% \mathrm{CI} 1,02-1.11), \mathrm{p}=0.001]$, and the limitation for activities of daily living [OR 4.65 (95\% CI 1.1 $-19.6), p=0.035] .70 \%$ of the decisions were recorded in the clinical history. The most frequently removed vital supports were: parenteral nutrition $(100 \%)$, vasopressors $(79 \%)$, enteral nutrition $(64 \%)$ and antimicrobials (52\%). Invasive mechanical ventilatory assistance was removed in $33 \%$ of the cases.

Conclusions: one third of patients die with LSTL. These types of decisions are associated with a poor quality of life, an older age and a longer stay in the ICU. One third of LTSV decisions are not registered. 


\section{Resumo}

Introdução: a limitação do emprego de terapias de suporte vital (LTSV) é uma prática habitual e eticamente aceitada. Esta decisão está compreendida no conceito de adequação do esforço terapêutico.

Objetivos: determinar a frequência, as normas de implementação e os fatores associados à LSTV nos pacientes falecidos em UCI (unidade de cuidados intensivos). Conhecer a frequência e as características do registro deste processo no prontuário do paciente.

Tipo de estudo: estudo observacional multicêntrico de corte transversal.

Escopo: foram analisados os prontuários dos pacientes de 8 UCIs polivalentes públicas e privadas do Uruguai.

Pacientes: foram incluídos todos os pacientes maiores de 18 anos falecidos em um período de 6 meses consecutivos em 2011.

Intervenções: análise das características dos pacientes pela revisão dos prontuários dos pacientes.

Resultados: analisamos 210 pacientes falecidos. Em 63 (30\%) pacientes foi registrada a LTSV. 75\% das decisões consistiam na retirada de medidas terapêuticas. Os fatores independentemente associados a LTSV foram: idade mais avançada [OR 1,04 (IC $95 \%$ $1,01-1,07), \mathrm{p}=0,001]$, maior permanência na UCI [OR 1,06 (IC 95\% 1,02 - 1,11), p = 0,001], e limitação para as atividades da vida diária [OR 4,65 (IC 95\% 1,1 -19,6), $\mathrm{p}=0,035] .70 \%$ das diretivas LTSV foram registradas no prontuário do paciente.

Os suportes vitais mais frequentemente retirados foram: nutrição parenteral (100\%), vasopressores (79\%), nutrição enteral (64\%) e antimicrobianos (52\%). A assistência ventilatória mecânica invasiva foi retirada em $33 \%$ dos casos.

Conclusões: um terço dos pacientes falece com LTSV. Este tipo de decisão está associada à má qualidade de vida previa, idade mais avançada e maior permanência em UCI. Um terço das decisões de LTSV não é registrado.

\section{Bibliografía}

1. Walling AM, Asch SM, Lorenz KA, Roth CP, Barry T, Kahn KL, et al. The quality of care provided to hospitalized patients at the end of life. Arch Intern Med 2010; 170: 1057-63.

2. Fumis RR, Deheinzelin D. Respiratory support withdrawal in intensive care units: families, physicians and nurses views on two hypothetical clinical scenarios. Crit Care 2010; 14(6):R235.

3. Rapin M, Le Gall JR. Palliative comfort therapy in critically ill patients. Bull Acad Nat Med 1979; 163:566-71.
4. Rapin M, Le Gall JR, Raphaël JC. Limitations of resuscitation. Acquis Med Recent 1981; 198:7-17.

5. Smedira NG, Evans BH, Grais LS, Cohen NH, Lo B, Cooke M, et al. Withholding and withdrawal of life support from the critically ill. N Eng J Med 1990; 322:309-15.

6. Bandrauk N, Downar J, Paunovic B. Withholding and withdrawing life- sustaining treatment: The Canadian Critical Care Society position paper. Can J Anaesth 2018; 65(1):105-22.

7. Yaguchi A, Truog RD, Curtis R, Luce JM, Levy MM, Mélot C, et al. International differences in end-of-life attitudes in the intensive care unit. Arch Intern Med 2005; 165:1970-5.

8. Cuttini M, Nadai M, Kaminski M, Hansen G, de Leeuw R, Lenoir S, et al. Endof-life decisions in neonatal intensive care: physicians' self reported practices in seven European countries. EURONIC Study Group. Lancet 2000; $355: 2112-8$.

9. Sprung CL, Eidelman LA. Worldwide similarities and differences in the forgoing of life-sustaining treatments. Intensive Care Med 1996; 22:1003-5.

10. Lobo SM, Simoni FHB, Jakob SM, Estella A, Vadi S, Bluethgen A, et al. Decision-making on withholding or withdrawing life-support in the ICU: a worldwide perspective. Chest 2017; 152:321-9.

11. Devictor DJ, Tissieres P, Gillis J, Robert Truog R. Intercontinental differences in end-of-life attitudes in the pediatric intensive care unit: results of a worldwide survey. Pediatr Crit Care Med 2008; 9:560-6.

12. Piva J, Lago P, Othero J, Garcia PC, Fiori R, Fiori H, et al. Evaluating end of life practices in ten Brazilian paediatric and adult intensive care units. J Med Ethics 2010; 36:344-8.

13. Moritz RD, Deicas A, Capalbo M, Forte DN, Kretzer LP, Lago P, et al. II Forum of the "End of Life Study Group of the Southern Cone of America": palliative care definitions, recommendations and integrated actions for intensive care and pediatric intensive care units. Rev Bras Ter Intensiva 2011; 23:24-9.

14. Azoulay E, Metnitz B, Sprung CL, Timsit JF, Lemaire F, Bauer P, et al. End-of-life practices in 282 intensive care units: data from the SAPS 3 database. Intensive Care Med 2009; 35:623-30.

15. Sprung CL, Carmel S, Sjokvist P, Baras M, Cohen SL, Maia P, et al. Attitudes of European physicians, nurses, patients, and families regarding end-of-life decisions: the ETHICATT study. Intensive Care Med 2007; 33:104-10.

16. Blazquez V, Rodríguez A, Sandiumenge A, Oliver E, Cancio B, Ibanez M, et al. Factors related to limitation of life support within $48 \mathrm{~h}$ of intensive care unit admission: a multicenter study. Med Intensiva 2018 May 7. doi: 10.1016/j.medin.2018.03.010 [Epub ahead of print].

17. Wynn A, Wise M, Wright MJ, Rafaat A, Wang YZ, Steeb G, et al. Accuracy of administrative and trauma registry databases. J Trauma 2001; 51:464-8. 
18. Spronk PE, Kuiper AV, Rommes JH, Korevaar JC, Schultz MJ. The practice of and documentation on withholding and withdrawing life support: a retrospective study in two Dutch intensive care units. Anesth Analg 2009; 109:841-6.

19. Mani RK, Mandal AK, Bal S, Javeri Y, Kumar R, Nama DK, et al. End-of-life decisions in an Indian intensive care unit. Intensive Care Med 2009; 35:1713-9.

20. Long AC, Muni S, Treece PD, Engelberg RA, Nielsen EL, Fitzpatrick AL, et al. Time to death after terminal withdra- wal of mechanical ventilation: specific respiratory and physiologic parameters may inform physician predictions. J Palliat Med 2015; 18:1040-7.

21. Gerstel E, Engelberg RA, Koepsell T, Curtis JR. Duration of withdrawal of life support in the intensive care unit and association with family satisfaction. Am J Respir Crit Care $2008 ; 178: 798-804$ 